\title{
Emerging Methods in Mechanical Behavior
}

\author{
B.L. Boyce $\cdot$ E.T. Lilleodden $\cdot$ X. Li
}

Published online: 6 August 2009

(C) Society for Experimental Mechanics 2009

Over the week of March 9-13, 2008, at the TMS Annual Meeting in New Orleans, Louisiana, a symposium was held on the topic of "Emerging Methods in Mechanical Behavior." While this topic is a common theme in the Society for Experimental Mechanics' meetings, this particular symposium was somewhat unusual because the host venue, TMS, is a materials society rather than a mechanics society. The TMS meeting holds symposia ranging from aluminum refining to phase transformations. A subset of materials scientists specialize in understanding the mechanical properties of materials. It is this group of researchers that the symposium had targeted: metallurgists, materials scientists, and mechanical engineers who are developing or employing new techniques to gain insight into the mechanical behavior of materials.

Materials Scientists typically come from a background that is strong in advanced characterization tools. For this reason, it is no surprise that this symposium on 'Emerging Methods in Mechanical Behavior' was laced with the quantitative application of characterization techniques in materials mechanics. Based on the contributions to this symposium, it is clear that diffraction

B.L. Boyce ( $\bowtie$, SEM member)

Sandia National Laboratories,

Albuquerque, NM, USA

e-mail: blboyce@sandia.gov

E.T. Lilleodden

GKSS Research Center,

Geesthacht, Germany

X. Li

University of South Carolina,

Columbia, SC 29208, USA techniques are expanding as a tool in experimental mechanics. While conventional x-ray diffraction has been used for decades as in the measurement of residual stresses, recently developed diffraction methods are becoming increasingly prevalent, including electron backscattered diffraction (also known as orientation imaging microscopy), synchrotron microdiffraction, and neutron diffraction. New microscopy techniques are also becoming prevalent. The dual-beam focused ion beam tool, which has emerged as a workhorse research tool in materials science over the past decade, permits microdissection, microwelding, ion channeling imaging, and many other creative new approaches to interrogation. Quantitative in-situ loading stages in scanning and transmission electron microscopies enable the connection between mechanical response and microscale evolution. In-situ imaging is providing valuable insight into nanoindentation and micropillar compression experiments. Another significant advance in quantitative experimental mechanics over the past decade has been the proliferation of digital image correlation (DIC) techniques. This surface deformation and strain mapping capability provides a tool for experimental mechanics that is loosely analogous to the advent of the finite element method for computational mechanics. Early applications of DIC were in the area of structural mechanics of lab-scale components. However, it is now clear that the DIC algorithm can also have applicability at the microscale through atomic force and scanning electron microscopies. Microscale DIC brings a powerful new tool to understand mechanical behavior at the microstructural scale. As shown in the collection of papers that are represented in this Special Issue of Experimental Mechanics, these emerging advanced characterization techniques are now providing crucial new insights into the mechanical behavior of materials. 